\title{
Effect on Osteogenesis of Cleaning Titanium Implants with Ozonated Water
}

\author{
Genki Yoshida $^{1,2)}$, Masahiko Ando ${ }^{1,2)}$, Yoshihiko Sugita ${ }^{3)}$, Hatsuhiko Maeda ${ }^{3)}$, Daisuke Kato ${ }^{1,2)}$, \\ Ryu Suzuki ${ }^{2)}$ and Hiroshi Murakami ${ }^{1,2)}$
}

\author{
${ }^{1)}$ Department of Gerodontology, School of Dentistry, Aichi Gakuin University, Nagoya, Japan \\ ${ }^{2}$ Division of Implant Dentistry, School of Dentistry, Aichi Gakuin University, Nagoya, Japan \\ ${ }^{3}$ Department of Oral Pathology, School of Dentistry, Aichi Gakuin University, Nagoya, Japan \\ (Accepted for publication, November 16, 2015)
}

\begin{abstract}
Carbon compounds accumulate over time on titanium surfaces. This is reported to reduce osseointegration of titanium implants in bone. Ozonated water was found to remove carbon compounds and to have a sufficient cleaning effect. The effects of the ozonated water on the titanium surface were evaluated by cell culture and mechanical tests. In vitro study, titanium disks (diameter $20 \mathrm{~mm}$, thickness $1 \mathrm{~mm}$, Grade 2) were treated with sulfuric acid. Disks in the control group were surface cleaned with distilled water, and disks in the $\mathrm{O}_{3}$ group were surface cleaned for $10 \mathrm{~min}$ with ozonated water. The experimental animals were male Sprague-Dawley (SD) rats. We tested for cell proliferation activity, alkaline phosphatase (ALP) activity, calcification and measured calcium concentrations. In vivo study, titanium implants (diameter $1 \mathrm{~mm}$, length 2 $\mathrm{mm}$, Grade 2) were treated with sulfuric acid. Control group samples were washed for $10 \mathrm{~min}$ with distilled water immediately before implantation. $\mathrm{O}_{3}$ group samples were washed for 10 min with ozonated water. We performed push-in testing using a mechanical testing machine. The femurs were removed at 2 and 4 weeks after implantation. Cell proliferation activity was higher in the $\mathrm{O}_{3}$ group than in the control group at $24 \mathrm{~h}$ after cell seeding. Similarly, ALP activity was higher in the $\mathrm{O}_{3}$ group than in the control group, indicating higher cell differentiation potential. Cell calcification was higher in the $\mathrm{O}_{3}$ group than in the control group at all measurement times. Maximum compressive stress was significantly higher in the $\mathrm{O}_{3}$ group compared with the control group at 2 weeks after implantation $(\mathrm{p}<0.05)$. At 4 weeks after implantation, no difference was found between the groups. Osseointegration time was decreased by cleaning titanium implants with ozonated water.
\end{abstract}

Key words: Carbon compounds, Osseointegration, Ozonated water, Osteoblast, Titanium surface

\section{Introduction}

Titanium is a lightweight metal with high corrosion resistance and good biocompatibility ${ }^{1}$. Therefore, it is widely used as a biomaterial in medicine and as a dental implant material in dentistry. The titanium surface and bone become fixed through the process of osseointegration, which results in close integration between the two at the light microscopic level ${ }^{2,3}$. This process of integration requires approximately 6 months for the maxilla and 3 months for the mandibular bone ${ }^{4)}$, which is a relatively long period of time when compared to prosthetic treatment such as dentures and bridges. Although improvement of the implant surface microstructure ${ }^{5-7)}$ and techniques such as immediate loading ${ }^{8)}$ and early loading ${ }^{9)}$ have been attempted to improve the process, cell adhesion in the early stage remains important to clinical outcome. Cell adhesion is affected by the physicochemical properties of

Correspondence to: Dr. Genki Yoshida, Department of Gerodontology, School of Dentistry, Aichi Gakuin University, 2-11 Suemori-Dori, Chikusa-ku, Nagoya, 464-8651 Japan; Tel: +81-52-751-7181; Fax: +8152-752-5990; Email: Mail: ag123d28@dpc.agu.ac.jp the titanium surface such as its microstructure, wettability, and adhesion with carbon compounds ${ }^{10-12)}$. In addition, there is still room for improvement in the ratio of the titanium surface area that is in close contact with the bone, which is currently 45-65 $\%^{13,14)}$. In the titanium implants now used in implant treatment, cell adhesion, proliferation, and differentiation decrease over time due to adhesion of carbon compounds to the titanium surface ${ }^{15,16)}$.

Ozone is used in various fields such as medicine, industry, and dairy farming for sterilization, deodorization, and bleaching ${ }^{17)}$ as well as in many areas of dentistry. When ozone dissolves in water, free radicals are formed due to the reaction with $\mathrm{OH}$ - ions, imparting ozonated water with strong oxidizing action ${ }^{18}$. When Murakami et al. ${ }^{19)}$, investigated the cleaning effect of ozonated water for removing carbon compounds from the titanium surface, the carbon compounds were found to be removed immediately after treatment.

In this study, to examine the influence of titanium treated with ozonated water on bone formation, we performed one in vitro and one in vivo study. In vitro study, osteoblast-like cells were applied 

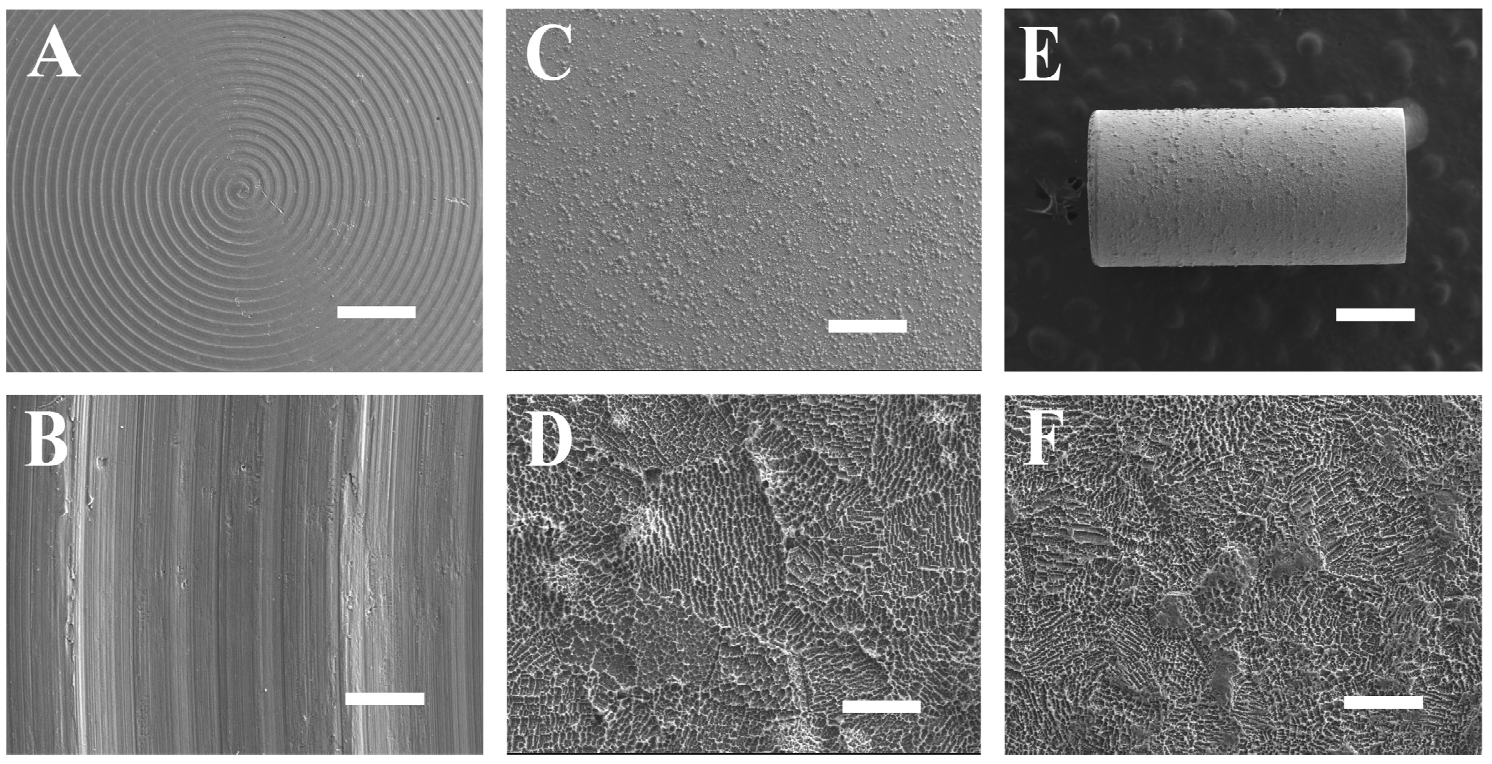

Figure 1. Low and high magnification SEM images of the titanium surface (upper row: low magnification, bar $=500 \mu \mathrm{m}$. lower row: high magnification, bar=20 $\mu \mathrm{m}$ ). Left column: Machined titanium surface (A, B). Center column: Acid treated surface $(C, D)$. Right column: Acid treated cylinder implant surface (E, F).

to pure titanium treated with ozonated water and cell proliferation, differentiation, and calcification were measured thereafter. In vivo study, pure titanium implants were placed in rat femurs, and mechanical strength was tested.

\section{Materials and Methods}

\section{Experimental animals}

The experimental animals were 5 male SD rats (Japan SLC, Hamamatsu, Japan) for in vitro study. Twenty-two 8-week old male SD rats were used for in vivo study. This study was approved by the Animal Research Committee of the Aichi Gakuin University School of Dentistry (approval number AGUD152, 217).

\section{Ozonated water production equipment}

An electrolytic ozonated water generator (Ozone Oral Irrigator, Ebara Jitsugyo Co, Tokyo, Japan) was used. The concentration of ozone was $5.0 \mathrm{mg} / 1\left(25^{\circ} \mathrm{C}\right)$ and its production rate was $40 \mathrm{ml} / \mathrm{min}^{19}$.

\section{Production and surface treatment of experimental sample}

The experimental samples were machined pure titanium disks (diameter $20 \mathrm{~mm}$, thickness $1 \mathrm{~mm}$, Grade 2, Nishimura Metal, Fukui, Japan) for in vitro study. Cylindrical titanium implants (diameter $1 \mathrm{~mm}$, length $2 \mathrm{~mm}$, Grade 2; Nishimura Metal, Fukui, Japan) were used as experimental samples for in vivo study. The surfaces of all samples were treated by thermal sulfuric acid processing for $75 \mathrm{~s}$ in a $67 \%$ sulfuric acid solution at $125^{\circ} \mathrm{C}$, after which ultrasonic cleansing was performed 3 times in pure water. Scanning electron microscopy images of the machined titanium surface and the titanium surface after acid processing are shown in Fig. 1.

After drying, all samples were kept in a dark room at normal temperature, and high-pressure steam sterilization was performed before the experiment. Samples were pre-treated for $10 \mathrm{~min}$ in either ozonated water $\left(\mathrm{O}_{3}\right.$ group) or distilled water (control group).

\section{Cell culture}

Marrow cells extracted from the femur of SD rat were cultured in medium containing 15\% FBS (Equitech-Bio, Kerrville, TX), $10^{-8} \mathrm{M}$ dexamethasone (Sigma, St. Louis, MO), $50 \mathrm{mg} / \mathrm{mL} \mathrm{L}-$ ascorbic acid 2 phosphoric acid (Sigma), $10 \mathrm{mM} \beta$ glycerophosphate (Sigma), antimicrobial and antifungal agents (100 U/ml penicillin $\mathrm{G}$ sodium, $100 \mathrm{mg} / \mathrm{ml}$ streptomycin sulfate, $250 \mathrm{ng} / \mathrm{ml}$ amphotericin B; Life Technologies, Carlsbad, CA), and $\alpha$-MEM (Life Technologies) with osteoblast inducer reagent. Cell culture took place in a $5 \% \mathrm{CO}_{2}, 37^{\circ} \mathrm{C}$ environment. As an initial culture, a $10-\mathrm{cm}$ diameter polystyrene culture plate (BD, Franklin Lakes, NJ) was seeded, and floating cells were removed on day 2 of culture and washed with phosphate-buffered saline (PBS). The culture medium was changed once every 3 days. On day 7 of culture, cells were collected for passaging after washing with PBS and incubating for 5 min with $0.25 \%$ trypsin-EDTA (Life Technologies). Centrifugal separation was performed in a $15-\mathrm{ml}$ tube (BD) at $1500 \mathrm{rpm}$ for $5 \mathrm{~min}$ and the supernatant solution was removed. The precipitate was then suspended in osteoblast inducer reagent and seeded into a stationary 12-well culture plate (Corning, Corning, NY) $\left(3 \times 10^{4}\right.$ cells/well $)$.

\section{Cell proliferation test}

For the cell proliferation test, a WST-8 kit (Cell Counting 
Genki Yoshida et al.: Effect on Osteogenesis of Titanium with Ozonated Water

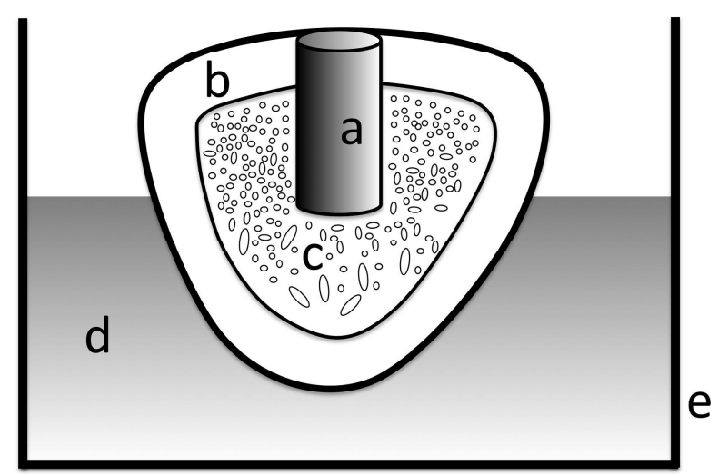

Figure 2. Schematic of implant placement in the rat femur. a: Cylinder titanium implant. b: Cortical. c: Medullary cavity. d: Resin. e: Metal frame.

Kit-8, Dojin Kagaku, Kumamoto, Japan) was used and cell numbers were measured. The titanium disks were moved to a new 12-well culture plate and washed twice with PBS. WST-8 reagent was added to $1000 \mu \mathrm{l}$ culture solution, incubated for $60 \mathrm{~min}$, and color reaction was performed. After that, the reagent was moved to a 96-well culture plate (BD), $450 \mathrm{~nm}$ absorbency was measured with an absorption spectrometer (Microplate Reader 680, BIORAD, Hercules, CA), and cell number change was measured as a relative value.

\section{Measurement of ALP activity}

ALP activity was measured on days 5 and 7 of culture using LabAssay ${ }^{\mathrm{TM}}$ ALP (Wako Pure Medicine Industries, Osaka, Japan). Titanium disks were moved to a new 12-well culture plate and washed twice with PBS. Then, $100 \mu 1$ buffer substrate was added and the plates were incubated for $10 \mathrm{~min}$ before color reaction was performed. Subsequently, $100 \mu 1$ of reaction liquid was moved to each well of a 96-well culture plate, and $405 \mathrm{~nm}$ absorbency was measured with an absorption spectrometer.

\section{Evaluation of calcification}

Alizarin Red staining was performed on days 7, 14, and 21 of culture to evaluate calcification. Titanium disks were moved to a new 12-well culture plate and fixed for $10 \mathrm{~min}$ in neutral buffered formalin solution (Muto Pure Chemicals, Tokyo, Japan). After washing in pure water, the titanium disks were stained with $\mathrm{pH}$ 4.1-4.3 adjusted Alizarin Red S stain and washed 4 times with pure water. The titanium disk was photographed with a digital camera, and the positive reaction area was quantified with image processing software (ImageJ, NIH, Bethesda, MD).

\section{Calcium determination test}

The calcium determination test was performed on days 14 and 21 of culture (Calcium Assay Kit, Cayman Chemical, Ann Arbor, MI). The calcium concentration on the titanium disks was quantified with observed data by absorption spectroscopy. The

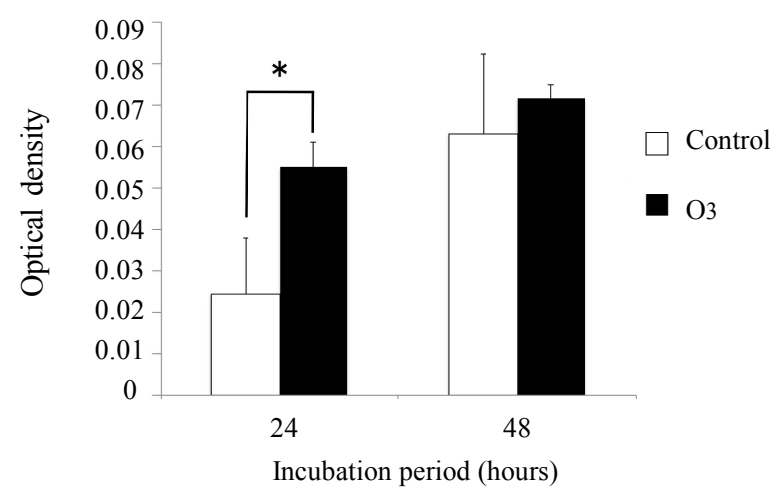

Figure 3. cell proliferation $(n=5)$

Absorption evaluated by the WST- 8 method. $* \mathrm{P}<0.05$.

titanium disks were moved to a new 12-well culture plate and soaked for $24 \mathrm{~h}$ in $0.5 \mathrm{M}$ hydrochloric acid to melt the surface calcium, and then $10 \mu \mathrm{L}$ samples were inserted into a 96-well culture plate. Calcium Detector R $1(100 \mu \mathrm{l})$ and Calcium Detector $\mathrm{R} 2(100 \mu \mathrm{l})$ mixture was then added and calcium concentration measured at $570 \mathrm{~nm}$ with an absorption spectrometer.

\section{Placement of cylindrical titanium implants}

After shaving, an incision was made in the femoral area of an anesthetized SD rat ( $2 \%$ isoflurane), and muscle and periosteum were avulsed and femoral bone exposed. A stoma was formed 10 $\mathrm{mm}$ from the kneecap with a $0.8 \mathrm{~mm}$ dental round bar (E0123, Dentsply Maillefer, Ballaigues, Switzerland), which was widened with a $\# 80 \mathrm{~K}$ file $(06026$, Ormco, Orange, CA) to form the bone fossa. A control group implant was placed in the right femur and an $\mathrm{O}_{3}$ group implant was placed in the left femur. The muscle and dermis were then sutured closed.

\section{Mechanical testing of osseointegration}

Push-in test ${ }^{20)}$ was used for mechanical testing. On days 14 and 28 after placement of the cylindrical titanium implant, 11 rats were humanely killed and the femur was extracted with ophthalmological scissors and fixed to a metal frame with autopolymerizing resin (GC, Tokyo, Japan) (Fig. 2). After that, a universal/tensile testing machine (EZ-S, Shimadzu Manufacturing, Kyoto, Japan) was used with a load applied in the long axis direction of the implant and compressive load (N) and pressure displacement $(\mu \mathrm{m})$ were measured.

\section{Statistical analysis}

F-test was used in all experiments. After verifying homoscedasticity, T-test analysis was performed. Statistical significance was set at $5 \%$.

\section{Results}

\section{Cell proliferation test}




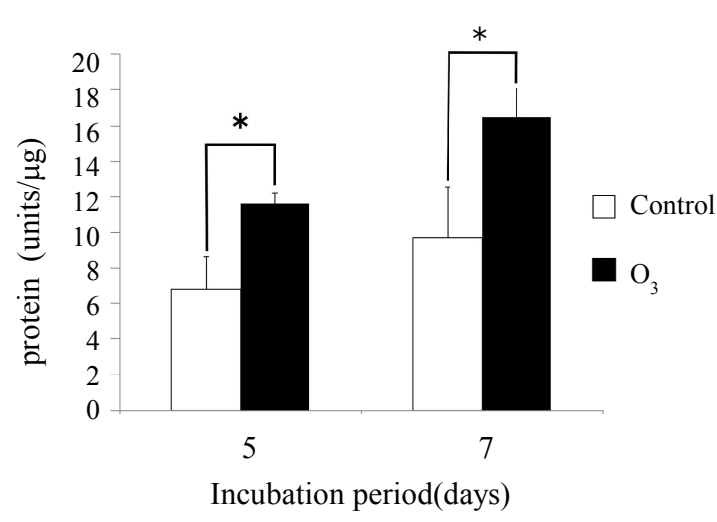

Figure 4: ALP activity $(\mathrm{n}=5)$

Protein mass calculated from absorption measured by the ALP activity test. $* \mathrm{P}<0.05$.

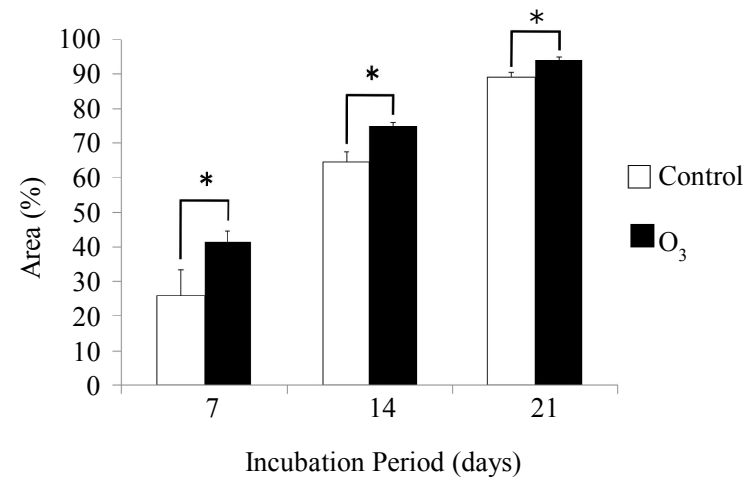

Figure 6. Evaluation of calcification erea $(n=5)$ Arizarin Red S staining image analysis results. ${ }^{*} \mathrm{P}<0.05$.

The results of the cell proliferation test are shown in Fig. 3. The graph in Fig. 2 shows absorption spectrometer value on the vertical axis and culture days on the horizontal axis. Values obtained from the absorption spectrometer after 24 and $48 \mathrm{~h}$ are expressed as increments. In the $24 \mathrm{~h}$ culture, the number of cells in the $\mathrm{O}_{3}$ group was significantly greater than in the control group $\left({ }^{*} \mathrm{P}<0.05\right)$. However, there was no significant difference in the 48 $\mathrm{h}$ culture.

\section{Measurement of ALP activity}

The results of ALP activity are shown in Fig. 4. In the graph in Fig. 4, the protein quantity calculated from absorption spectrometry is shown on the vertical axis and culture day is shown on the horizontal axis. Values obtained from the absorption spectrometer after 5 and 7 days are expressed as ALP activity increments. On culture days 5 and 7, ALP activity was significantly higher in the $\mathrm{O}_{3}$ group than in the Control group $(* \mathrm{P}<0.05)$.

\section{Evaluation of calcification}

Fig. 5 shows the results of Alizarin Red S staining (left: Control

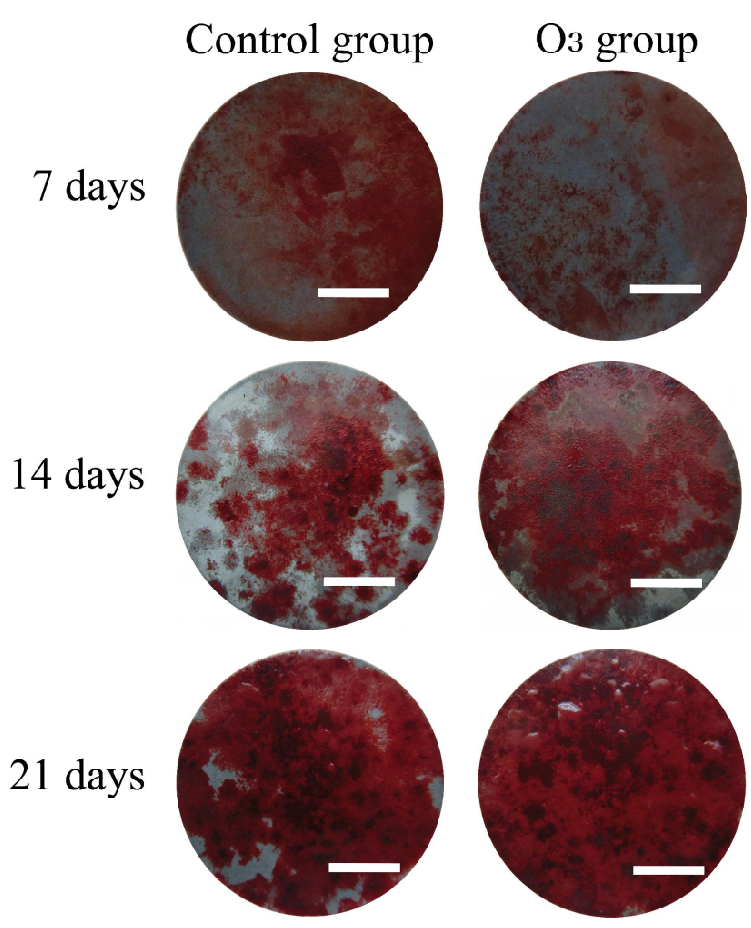

Figure 5. Arizarin Red S staining. $(\mathrm{bar}=5 \mathrm{~mm})$

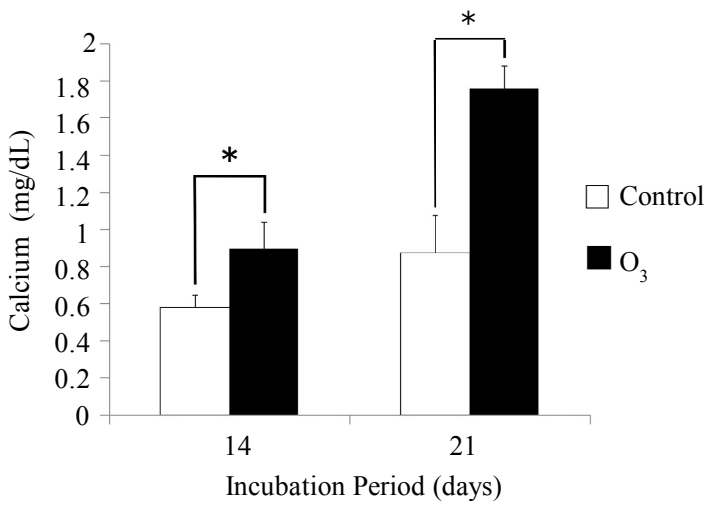

Figure 7. Calcium determination $(\mathrm{n}=5)$

Calcium concentration calculated from absorption spectra in the calcium determination test. ${ }^{*} \mathrm{P}<0.05$.

group, right: $\mathrm{O}_{3}$ group). On culture days 7, 14, and 21, the Alizarin Red S-stained positive reaction area was measured by image processing software (Fig. 6). The graph in Figure 6 shows the staining area ratio on the vertical axis and culture days on the horizontal axis. On culture days 7, 14, and 21, the staining area ratio was significantly higher in the $\mathrm{O}_{3}$ group compared to the control group. $(* \mathrm{P}<0.05)$

\section{Calcium determination test}

The results of calcium determination are shown in Fig. 7. The calcium quantity calculated from absorption spectrum is shown on the vertical axis and culture days on the horizontal axis. Values 


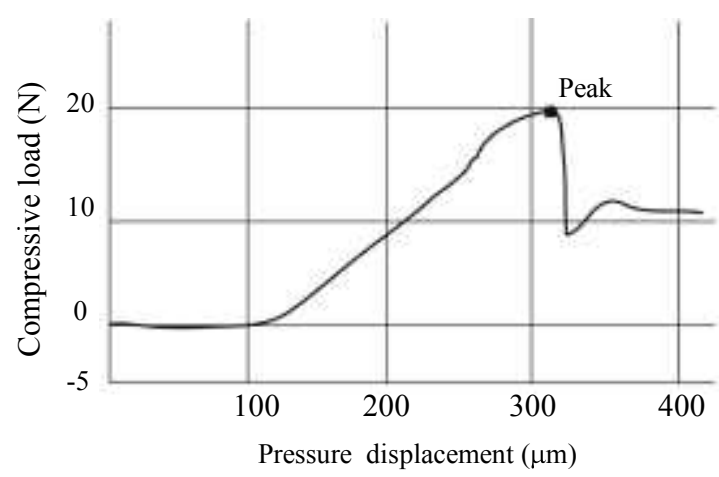

Figure 8. Example 1 of push-in test $\left(\mathrm{O}_{3}\right.$ group at day 28$)$.

obtained from the absorption spectrometer after 14 and 21 days are expressed as increases in calcium quantity. On culture days 14 and 21 , calcium quantity was significantly higher in the $\mathrm{O}_{3}$ group than in the control group $(* \mathrm{P}<0.05)$.

\section{Push-in test}

Fig. 8 shows an example of the mechanical testing of osseointegration. Compressive load is shown on the vertical axis and pressure displacement on the horizontal axis. In addition, the result is shown in Fig. 9. The vertical axis shows maximum compressive load value, and the horizontal axis shows duration in days since implantation. The compressive load on the cylindrical titanium implant increased gradually and decreased suddenly after the maximum load value was reached. On day 14 after implantation, the maximum compressive load value was significantly higher in the $\mathrm{O}_{3}$ group compared with the control group $\left({ }^{*} \mathrm{P}<0.05\right)$. However, at 28 days after implantation this value was higher in the $\mathrm{O}_{3}$ group but not significantly so.

\section{Discussion}

\section{Ozonated water}

Methods of generating ozonated water include bubbling or mixing ozone through distilled or purified water, as well as electrolysis ${ }^{21)}$. Here, following Murakami et al. ${ }^{19)}$, we used an electrolytic ozonated water generator (Ozone Oral Irrigator) that uses diamond electrodes. This method has a high efficiency of ozone generation and low production of ozone gas. When ozone molecules dissolve in water, they can remain as residual $\mathrm{O}_{3}$ or decompose into $\mathrm{OH}$ radicals, which are a more powerful oxidant than molecular ozone. There are two types of reactions involving soluble organic matter and ozone in the water: a direct reaction with ozone, and a free radical reaction with the formed $\mathrm{OH}$ radicals. Although lower temperature results in slower ozone autolysis and longer ozone half-life, in this study, the temperature was set at 25 ${ }^{\circ} \mathrm{C}$ for highest stability at room temperature. As $1 \mathrm{mg} / \mathrm{l}$ is sufficient for sterilization, and $4 \mathrm{mg} / \mathrm{l}$ is sufficient for virus inactivation ${ }^{18,22)}$, concentration was set at $5 \mathrm{mg} / \mathrm{L}$ to take the short half-life into consideration. In a preliminary experiment, treatment was

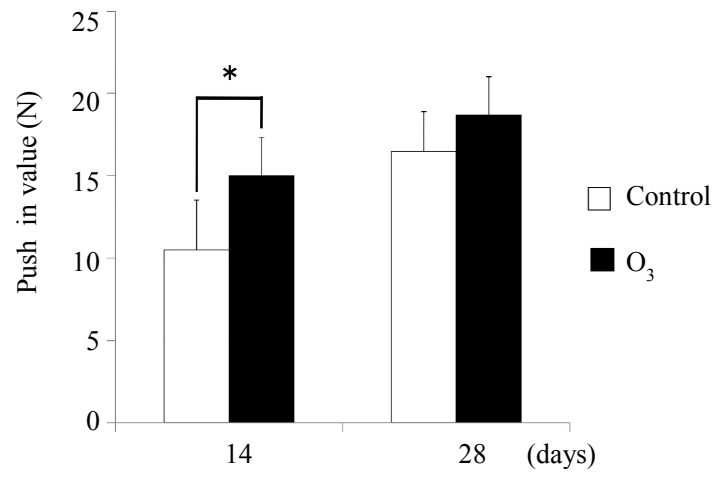

Figure 9. Push-in test $(\mathrm{n}=11)$

Maximum value of compressive load measured by mechanical testing. $* \mathrm{P}<0.05$.

performed for $1,3,5,7$ and $10 \mathrm{~min}$. At $10 \mathrm{~min}$ a significant difference was observed in cell proliferation. So for this study, treatment time was set at $10 \mathrm{~min}$.

\section{Sample production}

The as-produced titanium surface is hydrophilic, but with time carbon compounds adhere to the titanium surface, reducing the contact ratio and strength of the titanium/bone contact ${ }^{23,24)}$. The carbon compounds on the titanium surface increase sharply over 4 weeks after production, and then gradually over the next 3 months, while absorption of the proteins necessary for cell adhesion and osteogenesis falls notably. In light of this, the titanium disks in this study were used 3 months after initial processing.

\section{Cell culture of osteoblast-like cells}

Bone marrow contains mesenchymal stem cells, which have high differentiation potential. The osteoblast inducer reagent added to the culture solution functions to differentiate the mesenchymal stem cells into osteoblast-like cells. Mesenchymal cells can also differentiate into cartilage cells and adipose cells ${ }^{25-27)}$ but for this study, $10^{-8} \mathrm{M}$ dexamethasone, $50 \mathrm{mg} / \mathrm{ml} \mathrm{L}$-ascorbic acid, diphosphoric acid, and $10 \mathrm{mM} \beta$-glycerophosphate were added to the culture solution to specifically differentiate the bone marrow cells into osteoblast-like cells.

\section{Cell proliferation test}

In the 24-h culture, the number of cells was significantly higher in the $\mathrm{O}_{3}$ group than in the control group, but there was no significant difference in the 48 -h culture. This is likely due to the remarkable cell proliferation seen in the 24-h culture's proliferation curve during the logarithmic growth phase. It is expected that no difference would be seen at $48 \mathrm{~h}$, as the $\mathrm{O}_{3}$ group, in which the cells had reached confluence first, had stopped proliferating and the control group caught up to this level. 


\section{ALP activity}

ALP promotes the growth of hydroxyapatite crystals by breaking down pyrophosphoric acid and increasing local phosphoric acid concentration, which is characteristic of differentiation to osteoblast cells. On culture days 5, 7 ALP activity in the $\mathrm{O}_{3}$ group was significantly higher, as compared with the Control group.

\section{Evaluation of calcification}

Alizarin Red S staining confirmed that on culture days 7, 14 and 21 , the calcification area had increased significantly more in the $\mathrm{O}_{3}$ group compared with the control group. In the calcium determination test on culture days 14 and 21 in particular, the $\mathrm{O}_{3}$ group had a significantly higher value in comparison with the control group, at 1.5 times greater on day 14 and 1.9 times greater on day 21. However, the Alizarin Red S staining area ratio was 1.2 times greater on day 14 and 1.2 times greater on day 21 . The significant difference in area ratio was not large, but it suggests that the calcification node formed in the $\mathrm{O}_{3}$ group was sterically large. Carbon compounds were deposited on the titanium disks, which inhibits osteoblast activity ${ }^{16)}$ but by cleaning the disk surface with ozonated water, the carbon compounds were removed, as indicated by the promotion, proliferation, and differentiation of osteoblast-like cells.

\section{Push-in test}

Several studies have investigated the mechanical properties of the cylindrical titanium implants after placement in the rat femur $^{29,30)}$. Ogawa et al.'s push-in test has been proposed as one method of mechanical testing for verifying implant osseointegration $^{20)}$. Using the same method as Ogawa et al, this study tested implants of $1 \mathrm{~mm}$ in diameter and $2 \mathrm{~mm}$ in length, that were placed so as not to penetrate the opposite side (nonpenetration type). The mechanical test was performed on days 14 and 28 after implantation.

The compressive load of all samples was higher after placement of the cylindrical titanium implant and fell immediately after reaching the maximum value, which indicates total failure of osseointegration. The results of mechanical testing confirmed osseointegration in both groups. In both groups, there was a trend for the maximum value of compressive load to be greater after 28 days than after 14 days. On day 14 after placement of the cylindrical titanium implant, the maximum value of compressive load was significantly higher in the $\mathrm{O}_{3}$ group than in the control group. But on day 28, no significant difference was observed between the $\mathrm{O}_{3}$ and control group. Furthermore, the maximum value of compressive load for the $\mathrm{O}_{3}$ group on day 14 was close to the maximum value of the control group on day 28. This is thought to demonstrate that the $\mathrm{O}_{3}$ group was able to achieve osseointegration at an early stage. From the results above, ozonated water treatment allows early osseointegration, which suggests the possibility of a shorter treatment period.

From in vitro and in vivo study, it is thought that carbon compounds adhere to most titanium implants currently used in implant treatment ${ }^{13,14,31)}$. The carbon compounds adhering to the titanium surface at the time of placement of the implant are an important factor in determining early adhesion of osteoblast cells to the titanium surface ${ }^{16}$. However, it is not possible to control the accumulation of organic matter during production of titanium implants ${ }^{10,32)}$.

Other reported methods of managing carbon compound adhesion are ultraviolet treatment ${ }^{33)}$, storage in saline solution ${ }^{34)}$, and gamma-ray irradiation ${ }^{35)}$. Ultraviolet treatment is reported to cause physicochemical changes such as changing the titanium surface from hydrophobic to hydrophilic ${ }^{36)}$, causing the carbon atom ratio to decrease ${ }^{37,38)}$. However, this method has drawbacks in that decomposed carbon compounds remain on the surface, high-concentration ozone gas generated by the ultraviolet rays must be removed, and the apparatus is expensive. Gamma-ray irradiation of the titanium surface ${ }^{35}$ also decreases titanium surface carbon compounds and promotes osteoblast proliferation and differentiation, enabling early osseointegration. However, similar to ultraviolet treatment, the apparatus is expensive. Storage in saline solution immediately after manufacture of the titanium implant is reported to protect the pure titanium surface from contamination with organic matter and atmospheric carbon compounds and organic elements by keeping the implant hermetically sealed in saline ${ }^{34}$. However, most titanium implants produced today are kept in sterile cases after being exposed to the air. The cleaning effect of the ozonated water used in this study allows the by-products from decomposed carbon compound to be washed away, and the ozonated water not only promotes the decomposition reaction but also quickly returns to water after treatment, so it is non-toxic.

In conclusion, in this study, to examine cell adhesion and osseointegration in ozonated water-treated titanium surface, the proliferation, differentiation, and calcification potential of osteoblast-like cells was assessed by titanium surface cell culture testing and mechanical testing performed on cylindrical titanium implants after placement. The results showed that ozonated water treatment of the titanium surface promoted osteoblast-like cell proliferation and differentiation, and the improvement of mechanical strength suggested promotion of osteogenesis.

\section{Conflict of Interest}

The authors have declared that no COI exists.

\section{References}

1. Brunette DM, Tengvall P, Textor M and Thomsen P. Titanium 
Genki Yoshida et al.: Effect on Osteogenesis of Titanium with Ozonated Water

in Medicine, Springer (New York), 2001, pp 1-10

2. Nakamura HK, Butz F, Saruwatari L and Ogawa T. A role for proteoglycans in mineralized tissue-titanium adhesion. J Dent Res 86: 147-152, 2007

3. LeGeros RZ and Craig RG. Strategies to affect bone remodeling: osteointegration. J Bone Miner Res 8: 583596, 1993

4. Albrektsson T, Branemark P, Hansson $\mathrm{H}$ and Lindstrom J. Osseointegrated titanium implants. Requirements for ensuring a long-lasting, direct bone-to-implant anchorage in man Acta Orthop Scand 52: 155-170, 1981

5. Eckert SE, Choi YG, Sánchez AR and Koka S. Comparison of dental implant systems: quality of clinical evidence and prediction of 5-year survival. Int J Oral Maxillofac Implants 20: 406-415, 2005

6. Ogawa T, Ozawa S, Shih JH, Ryu KH, Sukotjo C, Yang JM and Nishimura I. Biomechanical evaluation of osseous implants having different surface topographies in rats. J Dent Res 79: 1857-1863, 2000

7. Sul Y, Johansson CB, Petronis S, Krozer A, Jeong Y, Wennerberg A and Albrektsson T. Characteristics of the surface oxides on turned and electrochemically oxidized pure titanium implants up to dielectric breakdown: the oxide thickness, micropore configurations, surface roughness, crystal structure and chemical composition. Biomaterials 23 : 491-501, 2002

8. Tarnow DP, Emtiaz S and Classi A. Immediate loading of threaded implants at stage 1 surgery in edentulous arches: ten consecutive case reports with 1 - to 5-year data. Int J Oral Maxillofac Implants, 12: 319-324, 1997

9. Szmukler-Moncler S, Salama H, Reingewirtz Y and Dubruille $\mathrm{JH}$. Timing of loading and effect of micromotion on bonedental implant interface: review of experimental literature. J Biomed Mater Res 43: 192-203, 1998

10. Weinlaender M, Kenney EB, Lekovic V, Beumer J, Moy PK and Lewis S. Histomorphometry of bone apposition around three types of endosseous dental implants. Int J Oral Maxillofac Implants 7: 491-496, 1992

11. Ogawa $T$ and Nishimura I. Different bone integration profiles of turned and acid-etched implants associated with modulated expression of extracellular matrix genes. Int $\mathbf{J}$ Oral Maxillofac Implants 18: 200-210, 2003

12. Zhao G, Schwartz Z, Wieland M, Rupp F, Geis-Gerstorfer J, Cochran DL and Boyan BD. High surface energy enhances cell response to titanium substrate microstructure. J Biomed Mater Res A 74: 49-58, 2005

13. Massaro C, Rotolo P, De Riccardis F, Milella E, Napoli A, Wieland M, Textor M, Spencer ND and Brunette DM. Comparative investigation of the surface properties of commercial titanium dental implants. Part I: chemical composition. J Mater Sci Mater Med 13: 535-548, 2002

14. Morra M, Cassinelli C, Bruzzone G, Carpi A, Di Santi G, Giardino R and Fini M. Surface chemistry effects of topographic modification of titanium dental implant surfaces: Surface analysis. Int J Oral Maxillofac Implants 18: 40-45, 2003

15. Att W, Horia N, Takeuchib M, Ouyangc J, Yangc Y, Anpob $\mathrm{M}$ and Ogawa T. Time-dependent degradation of titanium osteoconductivity: an implication of biological aging of implant materials. Biomaterials 30: 5352-5363, 2009

16. Hayashi R, Ueno T, Migita S, Tsutsumi Y, Doi H, Ogawa T, Hanawa $\mathrm{T}$ and Wakabayashi N. Hydrocarbon deposition attenuates osteoblast activity on titanium, J Dent Res 9: 698703, 2014

17. Rip GR. Century 21-Pregnant with ozone for applications. 15th OZONE WORLD CONGRESS, 1-19, 2001

18. Rip RG. Application of ozone for industrial wastewater treatment-A review. Ozone Sci Eng 18: 477-515, 1997

19. Murakami K, Li Y, Takeuchi K, Ueno A and Katou D. Effect of ozonated water cleaning on titanium surface carbon compound. Journal of JSOI 25: 171, 2012

20. Ogawa T, Ozawa S, Shih JH, Ryu KH, Sukotjo C, Yang JM and Nishimura I. Biomechanical evaluation of osseous implants having different surface topographies in rats. J Dent Res 79: 1857-1863, 2000

21. Velio B. Oxygen-Ozone Therapy Acritical Evaluation. Kluwer Academic Publishers, Boston, 2002, pp43-56

22. Viebahn HR. The Use of Ozone in Medicine, 3rd revised ed, transl. by Lee A, Hang KF, Medicina Biologica, 1999, pp1-148.

23. Att $\mathrm{W}$, Hori $\mathrm{N}$, Iwasa $\mathrm{F}$, Yamada $\mathrm{M}$, Ueno $\mathrm{T}$ and Ogawa $\mathrm{T}$. The effect of UV-photofunctionalization on the time-related bioactivity of titanium and chromium-cobalt alloys. Biomaterials 30: 4268-4276, 2009

24. Hori N, Att W, Ueno T, Sato N, Yamada M, Saruwatari L, Suzuki T and Ogawa T. Age-dependent degradation of the protein adsorption capacity of titanium. J Dent Res 88: 663667, 2009

25. Mark FP, Alastair MM, Stephen CB, Rama KJ, Douglas R, Joseph DM, Mark AM, Donald WS, Stewart C and Daniel RM. Multilineage potential of adult human mesenchymal stem cells. Science 284: 143-147, 1999

26. Colter DC, Sekiya I and Prockop DJ. Identification of a subpopulation of rapidly self-renewing and multipotential adult stem cells in colonies of human marrow stromal cells. Proc Natl Acad Sci USA 9: 7841-7845, 2001

27. Jones EA, Kinsey SE, English A, Jones RA, Straszynski L, Meredith DM, Markham AF, Jack A, Emery P and McGonagle D. Isolation and characterization of bone marrow multipotential mesenchymal progenitor cells. 
Arthritis Rheum 46: 3349-3360, 2002

28. Ohgushi H, Dohi Y, Katuda T, Tamai S, Tabata S and Suwa Y. In vitro bone formation by rat marrow cell culture. J Biomed Mater Res 32: 333-340, 1996

29. Yan J, Sun JF, Chu PK, Han Y and Zhang YM. Bone integration capability of a series of strontium-containing hydroxyapatite coatings formed by micro-arc oxidation. J Biomed Mater Res A 101: 2465-2480, 2013

30. Naka T and Yokose S. Application of laser-induced bone therapy by carbon dioxide laser irradiation in implant therapy. Int J Dent 2012: 1-8

31. Takeuchi M, Sakamoto K, Martra G, Coluccia S and Anpo M. Mechanism of photoinduced superhydrophilicity on the TiO2 photocatalyst surface. J Phys Chem B 109: 1542215428, 2005

32. Berglundh T, Abrahamsson I, Albouy JP and Lindhe J. Bone healing at implants with a fluoride-modified surface: An experimental study in dogs. Clin Oral Implants Res 18: 147152,2007

33. Aita H, Hori N, Takeuchi M Suzuki T, Yamada M, Anpo M and Ogawa $\mathrm{T}$. The effect of ultraviolet functionalization of titanium on integration with bone. Biomaterials 30: 10151025,2009
34. Buser D, Broggini N, Wieland M, Schenk RK, Denzer AJ, Cochran DL, Hoffmann B, Lussi A and Steinemann SG. Enhanced bone apposition to a chemically modified SLA titanium surface. J Dent Res 83: 529-533, 2004

35. Ueno T, Takeuchi M, Hori N, Iwasa F, Minamikawa H, Igarashi $\mathrm{Y}$, Anpo $\mathrm{M}$ and Ogawa T. Gamma ray treatment enhances bioactivity and osseointegration capability of titanium. J Biomed Mater Res B Appl Biomater 100: 22792287,2012

36. Suzuki T, Hori N, Att W, Kubo K, Iwasa F, Ueno T, Maeda $\mathrm{H}$ and Ogawa T. Ulutraviolet treatment Overcomes TimeRelated Degrading Bioactivity of Titanium. Tissue Eng Part A 5: 3679-3688, 2009

37. Yamada M, Miyauchi T, Yamamoto A, Iwasa F, Takeuchi M, Anpo M, Sakurai K, Baba K and Ogawa T. Enhancement of adhesion strength and cellular stiffness of osteoblasts on mirror-polished titanium surface by UV-photofunctionalization. Acta Biomater 6: 4578-4588, 2010

38. Iwasa F, Hori N, Ueno T, Minamikawa H, Yamada M and Ogawa T. Enhancement of osteoblast adhesion to UVphotofunctionalized titanium via an electrostatic mechanism. Biomaterials 31: 2717-2727, 2010 